\title{
Surfactant protein B polymorphisms, pulmonary function and COPD in 10,231 individuals
}

\author{
M. Bækvad-Hansen*, B.G. Nordestgaard**\# and M. Dahl*
}

ABSTRACT: The surfactant protein (SP)-B gene may influence chronic obstructive pulmonary disease (COPD) and, thus, personalised medicine. We tested whether functional polymorphisms in SP-B (rs1130866 (1580T>C), rs2077079 (-18A>C) and rs3024791 (-384G >A)) associate with reduced lung function and risk of COPD in the general population.

We genotyped 10,231 individuals from the general adult Danish population, and recorded spirometry and hospital admissions due to COPD. Because we previously found an association between the rare SP-B 121 ins 2 mutation and COPD among smokers, we stratified the analyses for smoking status.

None of the individual SP-B genotypes or genotype combinations were associated with reduced forced expiratory volume in 1 s (FEV 1 ) \% predicted, forced vital capacity (FVC) \% pred and FEV $1 /$ FVC overall or among smokers $(p=0.25-0.99)$. The odds ratio for spirometrically defined COPD did not differ from 1.0 for any of the SP-B genotypes or genotype combinations overall or among smokers $(p=0.17-0.78)$. Similar results were obtained for hospitalisation due to COPD $(p=0.07-$ 0.93); we could exclude overall hazard ratios for heterozygotes of 1.18-1.21 and for homozygotes of 1.25-1.57 or larger for all three polymorphisms.

In conclusion, the functional rs1130866, rs2077079 and rs3024791 polymorphisms in the SP-B gene are not associated with reduced lung function or risk of COPD, making it unlikely that these variants will be useful in personalised medicine.

KEYWORDS: Asthma, chronic obstructive pulmonary disease, genetics, interstitial lung disease, lung cancer, pneumonia

hronic obstructive pulmonary disease (COPD) is one of the leading causes of death worldwide and the number of deaths due to COPD is expected to rise in the future [1]. Smoking is the main risk factor for development of COPD; however, far from all smokers develop the disease. This suggests that other factors, such as genetic background, may play a role in susceptibility to COPD and, thus, be useful in personalised medicine. A variety of genes have been linked with risk of COPD, among these the surfactant protein (SP) genes [2-4].

SPs are essential components of the lung surfactant layer, which covers the terminal airways. The lung surfactant consists of phospholipids, cholesterol and proteins, and is primarily produced by alveolar type II cells [5, 6]. It forms a thin lipid layer on the surface of alveoli, which reduces surface tension and prevents alveoli from collapse during expiration. Upon exocytosis from alveolar type II cells, surfactant initially exists as multilayered vesicular structures in the epithelial lining fluid. From these structures, it spreads to the surface as alveoli are extended and compressed during breathing.

SP-B is one of the four known SPs in humans. It is important for the formation of lamellar bodies and correct assembly of the surfactant layer [7]. Lack of SP-B cause fatal respiratory distress syndrome in newborns [8], and genetic markers in and around the $S P-B$ gene have been associated with a spectrum of pulmonary diseases, including COPD [2-4, 9-13]. Three functional polymorphisms in $S P-B$, rs1130866, rs2077079 and rs3024791, have been associated with risk and/or severity of COPD. rs1130866 abolishes an $\mathrm{N}$-linked glycosylation site in $S P-B$, while the two

This article has supplementary material available from www.erj.ersjournals.com

AFFILIATIONS

*Dept of Clinical Biochemistry, Herlev Hospital, and

\#The Copenhagen City Heart Study, Bispebjerg Hospital, Copenhagen University Hospital, Faculty of Health Sciences, University of Copenhagen, Copenhagen, Denmark.

CORRESPONDENCE

M. Dahl

Dept of Clinical Biochemistry Herlev Hospital

Copenhagen University Hospital

Herlev Ringvej 75

DK-2730 Herlev

Denmark

E-mail: mordah02@heh.regionh.dk

Received

Feb 162010

Accepted after revision:

July 182010

First published online:

Aug 062010 


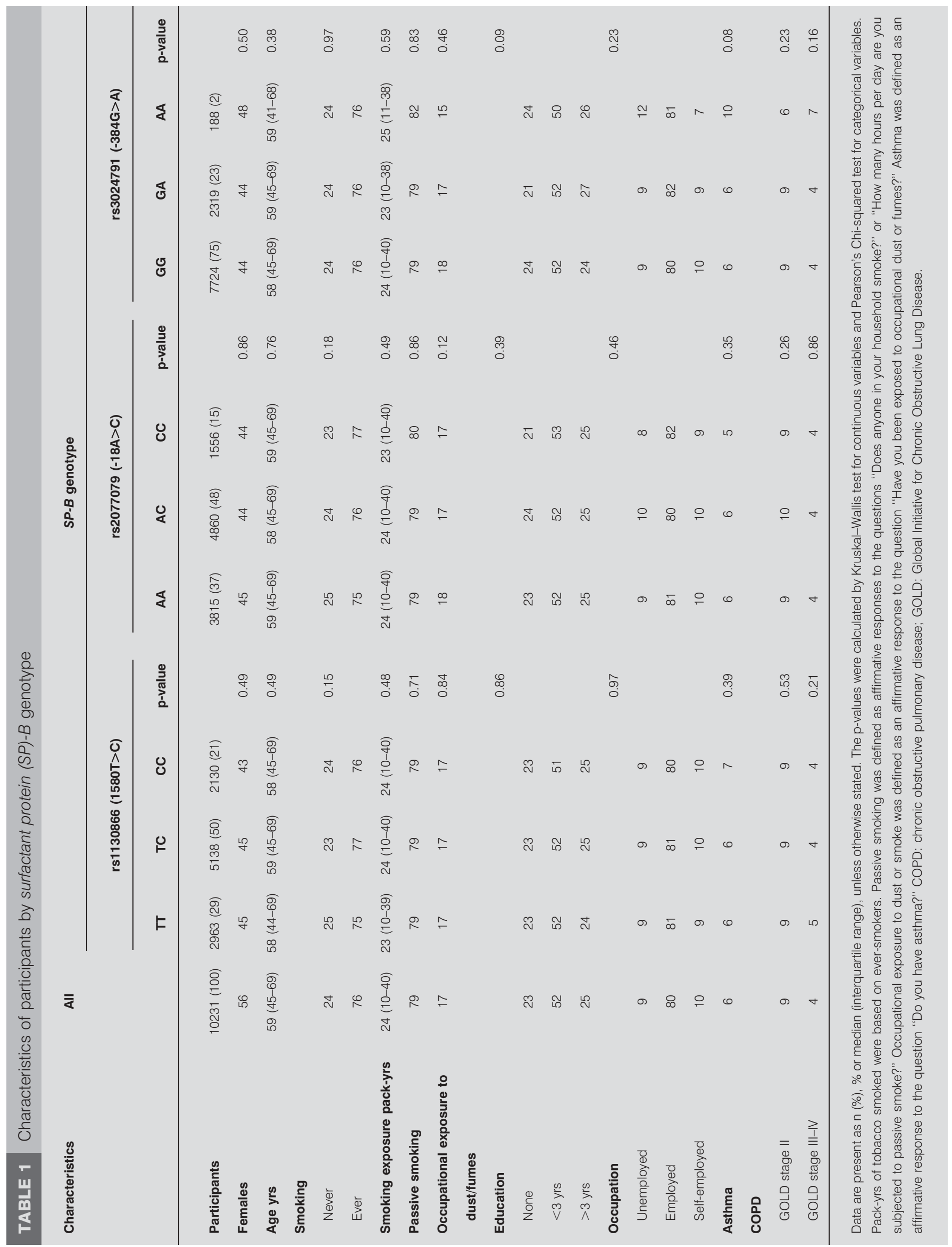


TABLE 2 Patients with chronic obstructive pulmonary disease (COPD) according to sex and age

\begin{tabular}{|c|c|c|c|c|c|}
\hline & \multirow[t]{2}{*}{ All } & \multicolumn{2}{|c|}{ Females } & \multicolumn{2}{|c|}{ Males } \\
\hline & & Age $<67$ yrs & Age $\geqslant 67$ yrs & Age $<67$ yrs & Age $\geqslant 67$ yrs \\
\hline Spirometrically defined COPD & $1327(100)$ & $296(22)$ & $309(23)$ & $340(26)$ & $382(29)$ \\
\hline COPD hospitalisation & $1186(100)$ & $347(29)$ & $297(25)$ & $278(23)$ & $264(22)$ \\
\hline Any COPD & $1936(100)$ & $501(26)$ & $462(24)$ & $492(25)$ & $481(25)$ \\
\hline
\end{tabular}

promoter polymorphisms, rs2077079 and rs3024791, alter $S P-B$ transcription levels [3, 14-18].

We hypothesised that the three common functional polymorphisms (rs1130866, rs2077079 and rs3024791) in the SP-B gene are associated with reduced lung function and increased risk of COPD in the general population. To test this hypothesis, we genotyped 10,231 individuals from the general adult Danish population, and recorded spirometry and hospital admissions due to COPD. We calculated odds and hazard ratios (HRs) to assess risk of COPD according to $S P-B$ genotype, and we used power calculation to estimate the maximal risk of COPD we could potentially have overlooked.

\section{MATERIALS AND METHODS \\ Subjects}

We genotyped 10,231 individuals from the Copenhagen City Heart Study, a prospective general population study of individuals selected from the Central Population Register Code to reflect the adult Danish population aged 20 to $\geqslant 80$ yrs [19]. The Copenhagen City Heart Study was initiated in 1976-1978 with follow-up examinations in 1981-1983, 1991-1994 and 2001-2003. DNA for genotyping was isolated from participants attending the 1991-1994 and/or 2001-2003 examinations. The study was approved by the local ethical committee: study numbers 100.2039/91 and 01-421/94, Copenhagen and Frederiksberg committee. All participants gave written informed consent. All participants were white subjects of Danish descent

\section{Pulmonary function testing and COPD diagnoses}

Forced expiratory volume in $1 \mathrm{~s}$ (FEV1) and forced vital capacity (FVC) were determined with a dry-wedge spirometer (Vitalograph, Maids Moreton, UK). Each spirometry was performed in triplicate and results were accepted only if the variation between the two best measurements was $<5 \%$. The best results were used for calculation of FEV1 \% predicted and FVC \% pred using multiple regressions on never-smokers for males and females separately, with age and height as covariates $[19,20]$. Spirometrically defined COPD was taken as FEV1/FVC $<0.7$ and FEV1 $<80 \%$ pred [4]. If individuals with asthma were excluded from this definition and/or individuals with Global Initiative for Chronic Obstructive Lung Disease (GOLD) stage I (FEV1/FVC $<0.7$ and FEV1 $>80 \%$ pred) were included, the results were similar to those presented. Information on hospitalisation due to COPD (International Classification of Diseases (ICD)-8 491-492 and ICD-10 J41-J44) was collected in the national Danish Patient Registry, which covers all hospitals in Denmark from 1976 to 2009.

\section{Other pulmonary diseases}

Asthma was defined as an affirmative response to the question "Do you have asthma?" Chronic bronchitis was defined as an affirmative response to the question "Do you bring up phlegm at least 3 months continuously every year?" Information on pneumonia, interstitial lung disease and lung cancer was collected in the national Danish Patient Registry and the Danish Cancer Registry. Pneumonia (ICD-8 480-486 and ICD$10 \mathrm{~J} 12-\mathrm{J} 18)$, interstitial lung disease (ICD-10 J84) and lung cancer (ICD-7 162.0-2, 162.4-7, 163.0, 164.0, 199.2, 462.1-4, 464.4, 962.1-2, 962.4-6, 963.0 and 964.0, and ICD-10 C33-C34, C37-C38 and D02.1-2).

\section{Genotyping}

Genomic DNA was isolated from frozen whole blood (Qiagen, Hilden, Germany). The genotype analysis was performed in 2007 using the ABI PRISM ${ }_{\circledast}$ 7900HT Sequence Detection System (Applied Biosystems Inc., Foster City, CA, USA). Primers and probes for the TaqMan assays are listed in supplementary table 1 . The TaqMan analysis was validated by sequencing of a subsample of the participants.

\section{Statistical analysis}

Statistical analyses were performed using STATA SE version 10.0 (StataCorp, College Station, TX, USA). A two-sided p-value of $<0.05$ was considered significant. From the three polymorphisms, we generated all possible genotype combinations. We used the Kruskal-Wallis test and Pearson's Chi-squared test for differences in characteristics between $S P-B$ genotypes (table 1). Main effects of $S P-B$ genotypes in predicting FEV1\% pred, FVC $\%$ pred and FEV1/FVC were examined using the test for trends, while associations of $S P-B$ genotype combinations with lung function were tested in ANOVA models. Odds ratios for COPD on spirometry by $S P-B$ genotype or genotype combination were from logistic regression models including age, sex, smoking exposure in pack-yrs, passive smoking, occupational dust and fumes, education, and occupation. HRs for COPD hospitalisation during $\leqslant 33$ yrs of follow-up by $S P-B$ genotype or genotype combination were determined by Cox regression models with age as the timescale, adjusted for age, sex, pack-yrs, passive smoking, occupational dust and fumes, education, and occupation. We used NCSS-PASS (NCSS, Kaysville, UT, USA) to 
calculate the low and high odds and HRs, which we had $90 \%$ power to exclude at two-sided p-values $<0.05$. Linkage disequilibrium between polymorphisms was estimated by Lewontins D' using STATA's pwld function.

\section{RESULTS}

Characteristics of the participants are shown in table 1. There were no differences in sex, age, smoking status or pack-yrs of tobacco smoked for any of the $S P-B$ genotypes. The distributions of $S P-B$ genotypes for the three polymorphisms were in HardyWeinberg equilibrium (rs1130866: $p=0.26$; rs2077079: $p=0.96$; rs3024791: $p=0.32$ ). Pair-wise linkage disequilibrium for the three polymorphisms was determined by D'. rs2077079 and rs3024791 were in tight linkage disequilibrium $\left(\mathrm{D}^{\prime}=0.97\right)$, whereas there was low linkage disequlibrium between rs1130866 and either rs2077079 or rs3024791 ( $\mathrm{D}^{\prime}=0.21$ and 0.18 , respectively). The relationship between spirometrically defined COPD and COPD hospitalisation, according to sex and age, is presented in table 2.
The proportion of males tended to be higher among those with spirometrically defined COPD and age $>66$ yrs, while the proportion of females tended to be higher among those with COPD hospitalisation and age $<67$ yrs. The proportions of females and males were similar among individuals with any COPD, i.e. spirometrically defined COPD or COPD hospitalisation.

\section{Lung function}

None of the individual $S P-B$ genotypes or genotype combinations were associated with reduced FEV1 \% pred, FVC \% pred or FEV1/FVC ( $p=0.34-0.94$; fig. 1$)$. When stratifying for smoking status, the $\mathrm{p}$-value for $S P-B$ genotype combinations reached significance for $\mathrm{FEV} 1 \%$ pred and $\mathrm{FVC} \%$ pred among never-smokers (fig. 2). On post hoc analysis, the SP-B TCAAGG and TCCCGG versus TCACGG genotypes were associated with increased FEV1 \% pred ( $p=0.02$ and $p=0.01$, respectively) and FVC $\%$ pred $(\mathrm{p}=0.02$ and $\mathrm{p}=0.003$, respectively) among neversmokers. Conversely the SP-B CCCCGA versus TCACGG

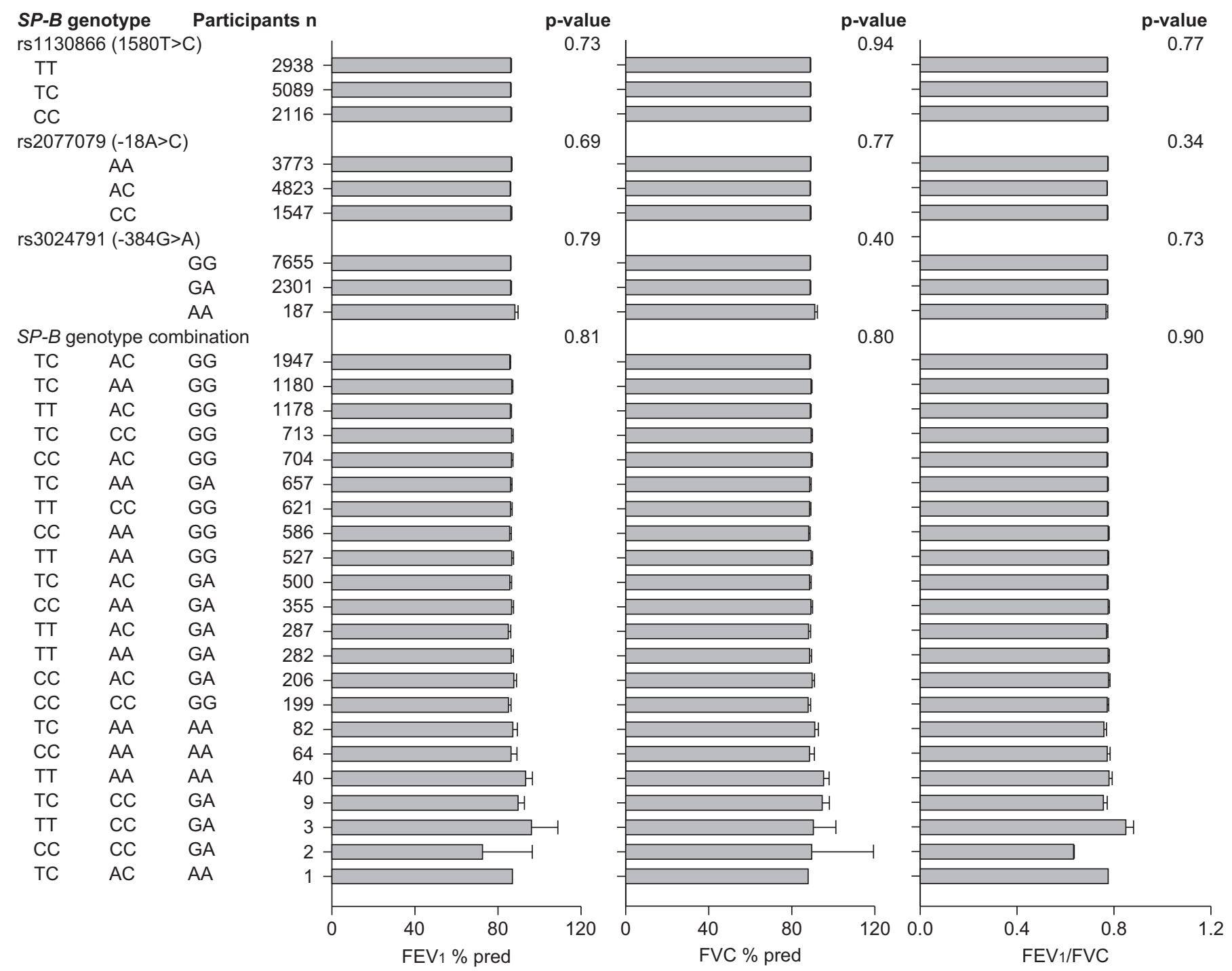

FIGURE 1. Lung function according to surfactant protein (SP)-B genotypes and genotype combinations. Data are presented as mean \pm SE. p-values for $S P$ - $B$ genotypes and genotype combinations were by test for trends and ANOVA, respectively. Numbers are slightly less than all individuals genotyped, as not all participants had spirometry performed. FEV1: forced expiratory volume in $1 \mathrm{~s} ; \%$ pred: \% predicted; FVC: forced vital capacity. 

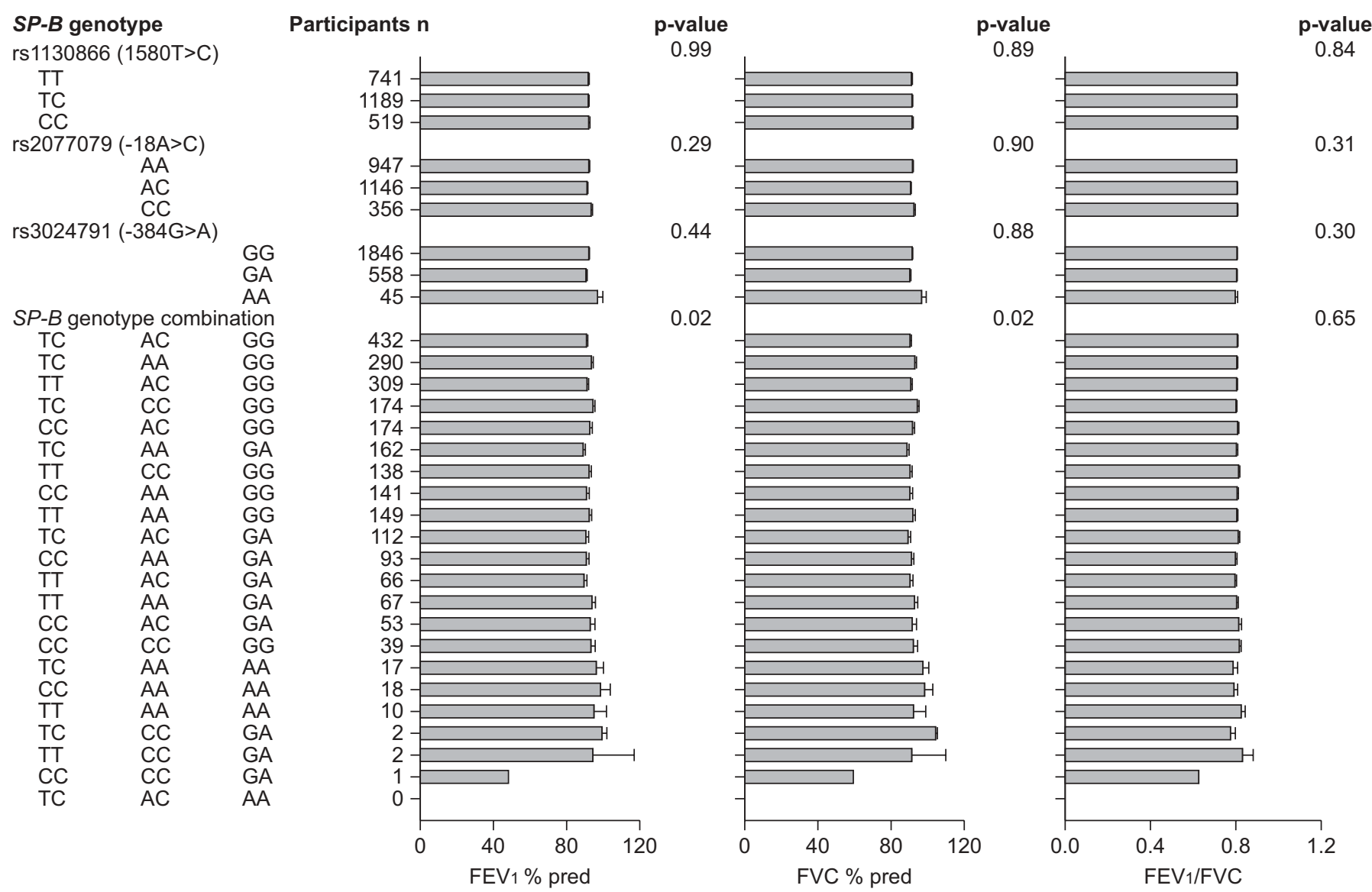

FIGURE 2. Lung function according to surfactant protein (SP)-B genotypes and genotype combinations among never-smokers. Data are presented as mean \pm SE. p-values for SP-B genotypes and genotype combinations were by test for trends and ANOVA, respectively. Numbers are slightly less than all individuals genotyped, as not all participants had spirometry performed. FEV1: forced expiratory volume in $1 \mathrm{~s}$; \% pred: \% predicted; FVC: forced vital capacity.

genotype was associated with reduced FEV1 \% pred $(\mathrm{p}=0.007)$, FVC $\%$ pred $(p=0.04)$ and FEV1/FVC $(p=0.01)$ among neversmokers. These results are not biologically plausible and they could not be confirmed when testing spirometrically defined COPD or COPD hospitalisation. Among ever-smokers, none of the individual $S P-B$ genotypes or genotype combinations were associated with reduced FEV1 \% pred, FVC \% pred and FEV1/ FVC ( $p=0.25-0.99$; online supplementary fig. 1$)$.

\section{Risk of COPD}

The odds ratio for spirometrically defined COPD did not differ from 1.0 for any $S P-B$ genotype or genotype combination, nor did the risk for COPD hospitalisation (fig. 3). We had $90 \%$ statistical power to exclude odds ratios for spirometrically defined COPD for heterozygotes of 1.22-1.24 and for rare homozygotes of 1.30-1.96 or larger for all three polymorphisms (fig. 3). Likewise, we had $90 \%$ statistical power to exclude HRs for COPD hospitalisation for heterozygotes of 1.18-1.21 and for rare homozygotes of $1.25-1.57$ or larger for all three polymorphisms.

When stratifying for smoking status, the p-value for the rs1130866 polymorphism reached significance for COPD hospitalisation among never-smokers (fig. 4); however, none of the individual rs1130866 CC and TC genotypes differed significantly from 1.0, and the result could not be confirmed when analysing spirometrically defined COPD. The risk for spirometrically defined COPD was increased in SP-B TTACGG and TCAAGA versus TCACGG among never-smokers (OR 2.5 (95\% CI 1.2-5.5) and OR 2.7 (95\% CI 1.1-6.7), respectively), and for COPD hospitalisation in SP-B CCAAAA versus TCACGG among never-smokers (HR 8.6 (95\% CI 1.7-42.6)). None of these results could be confirmed when analysing the other COPD outcome of the study or when analysing the lung function. We, therefore, interpret these findings as likely spurious results.

Because the rs1130866 and the rs2077079 polymorphisms have been associated with severity of COPD and COPD exacerbations $[3,16]$, we examined the prevalence of COPD GOLD stages by $S P-B$ genotype. The prevalence of GOLD stages II and III-IV did not differ by any of the $S P-B$ genotypes $(\mathrm{p}=0.16-0.86)$.

\section{Risk of other lung diseases}

We also tested whether the three $S P-B$ polymorphisms were associated with asthma, interstitial lung disease, pneumonia, chronic bronchitis or lung cancer (online supplementary table 2). None of the $S P-B$ genotypes was associated with any of these lung diseases, except the rs3024791 AA genotype, which showed a 1.8-fold increased risk for asthma over GG. 
Spirometrically defined COPD

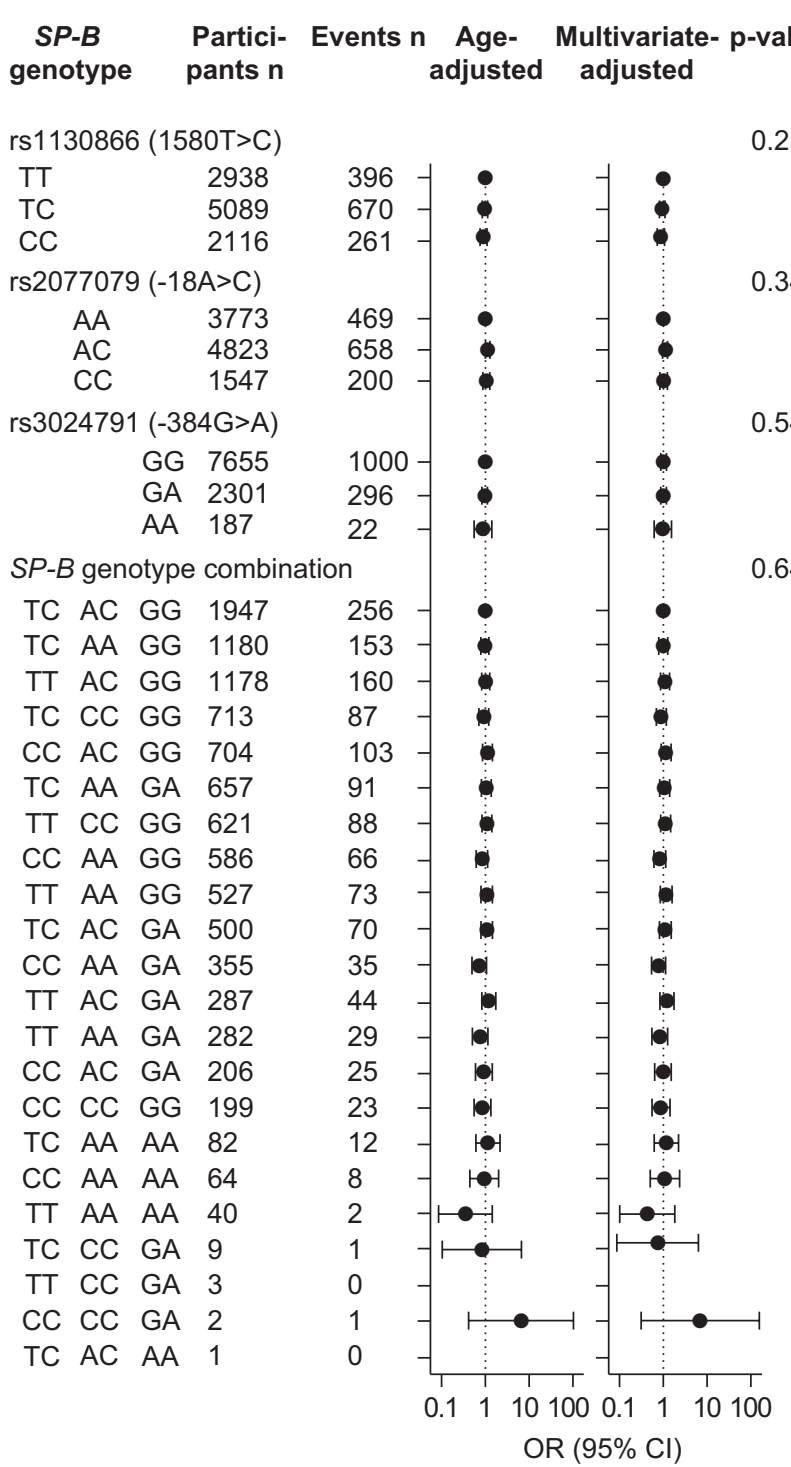

COPD hospitalisation

90\% power Partici- Events n Age- Multivariate- p-value $90 \%$ power Low High pants $n$ adjusted adjusted

Low High

0.25

$\begin{array}{lll}0.79 & 1.24 & 2963 \\ 0.75 & 1.30 & 2138 \\ & & \\ & & 3815 \\ 0.81 & 1.22 & 4860 \\ 0.73 & 1.33 & 1556 \\ & & \\ & & 7724 \\ 0.79 & 1.24 & 2319 \\ 0.38 & 1.96 & 188\end{array}$

0.64

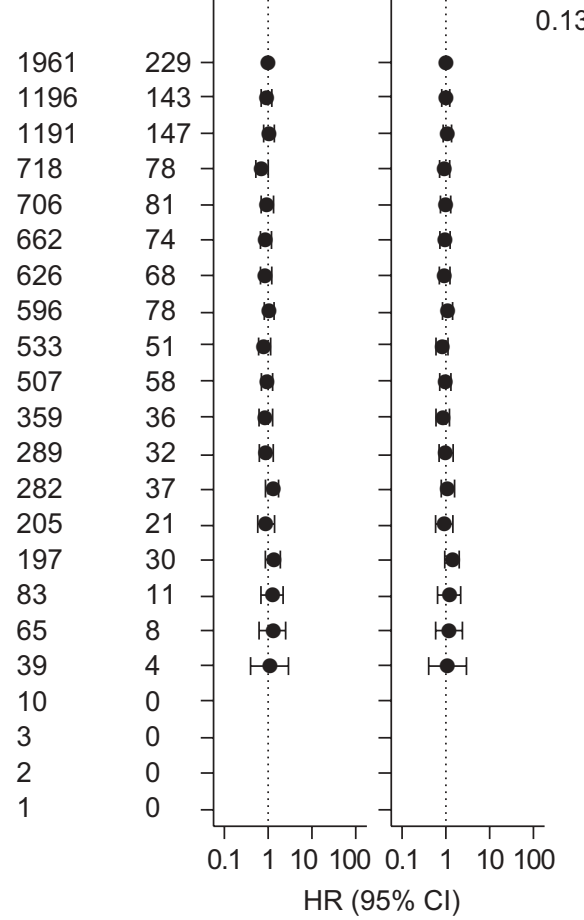

0.72

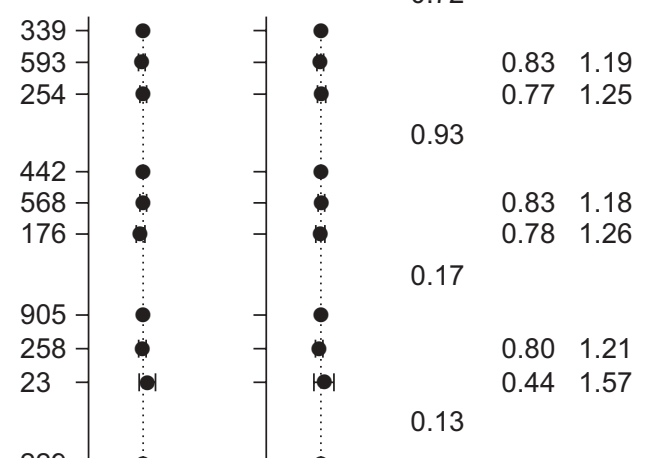

FIGURE 3. Risk of chronic obstructive pulmonary disease (COPD) according to surfactant protein (SP)-B genotypes and genotype combinations. Spirometrically defined COPD: forced expiratory volume in $1 \mathrm{~s}$ (FEV 1 )/forced vital capacity < 0.7 and FEV $1<80 \%$ predicted. COPD hospitalisation: International Classification of Diseases (ICD)-8 491-492 or ICD-10 J41-J44. Odds ratios for spirometrically defined COPD are by logistic regression. Hazard ratios (HR) for COPD hospitalisation are by Cox regression. Multivariate adjusted models allowed for age, sex, pack-yrs, passive smoking, occupational dust and fumes, education, and occupation. p-values for SP-B genotypes and genotype combinations were by test for trends and ANOVA, respectively. The 90\% power indicates the odds ratios that can be detected in this study at two-sided $p<0.05$. Numbers for the analysis of spirometrically defined COPD are slightly less than for COPD hospitalisation, because not all individuals had spirometry performed.

\section{DISCUSSION}

Genetic variation in $S P-B$ has been linked to COPD in several association studies $[3,9,16,18]$ and, thus, could have importance for the development of COPD in the general population. We determined whether three functional polymorphisms in $S P-B$ were associated with poor lung function and COPD in a large homogenous Danish population sample. We found, with significant power, that these polymorphisms were not associated with lung function or risk of COPD overall or among smokers. This makes it unlikely that the genetic variants can be used clinically to assess risk of COPD or to identify COPD subgroups for tailored therapy.
The C allele of the rs113086 polymorphism has previously, in studies with $<1,000$ participants, been associated with increased risk of COPD, severity of airway obstruction and disease exacerbation in COPD patients $[3,14,16,18]$. In contrast, the present population-based study with $>10,000$ participants indicated that the rs1130866 polymorphism does not affect risk of COPD, severity of COPD or risk of any other additional pulmonary disorder overall or among smokers. We did find a trend towards lower risk of COPD hospitalisation among neversmokers. However, this result could not be confirmed when analysing spirometrically defined COPD or lung function. We, therefore, interpret this as a likely spurious finding. Our data 
Spirometrically defined COPD

COPD hospitalisation

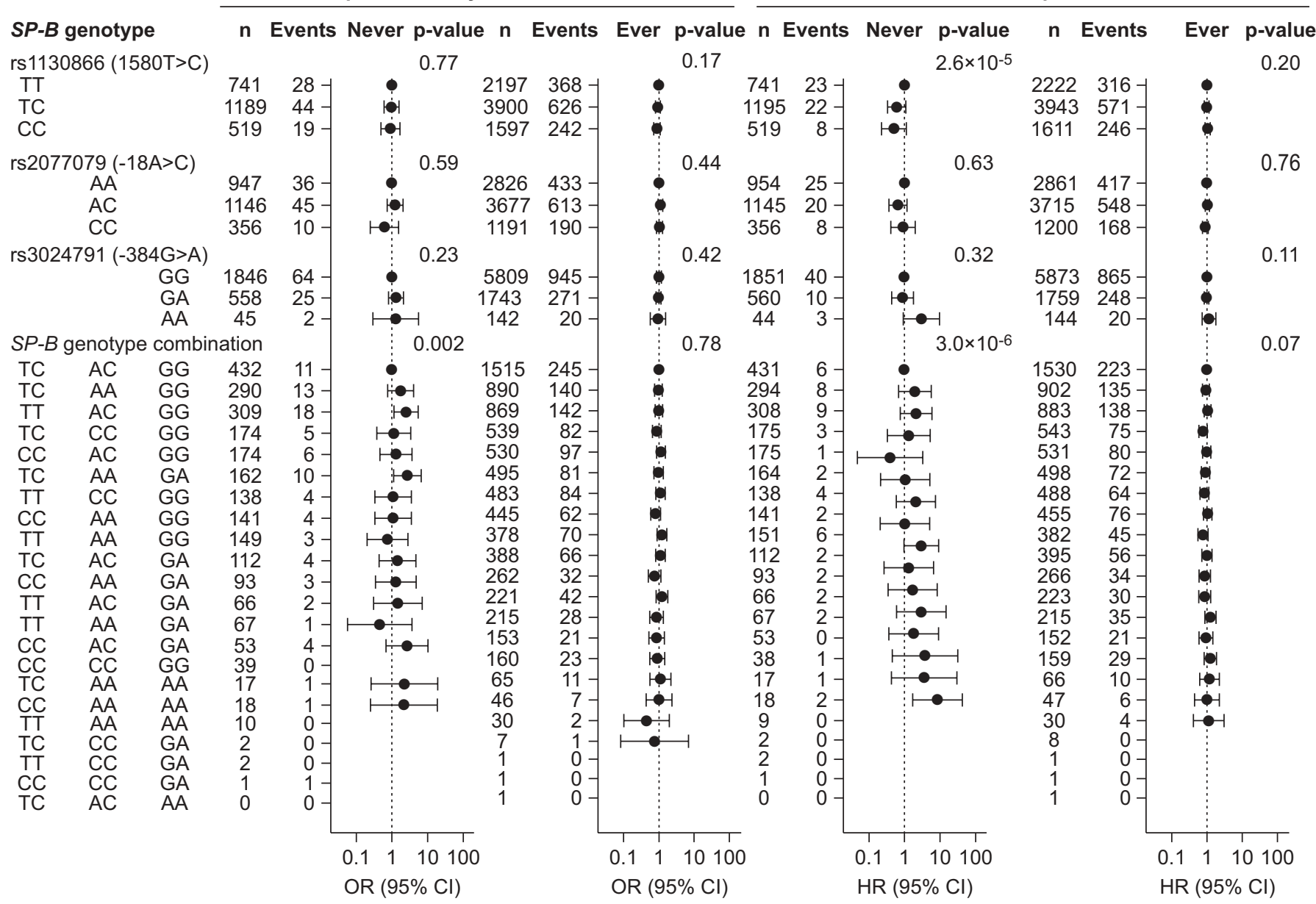

FIGURE 4. Risk of chronic obstructive pulmonary disease (COPD) according to surfactant protein (SP)-B genotypes and genotype combinations, stratified by smoking status. Ever-smokers: current and former smokers. Spirometrically defined COPD: forced expiratory volume in $1 \mathrm{~s}$ (FEV1)/forced vital capacity $<0.7$ and $\mathrm{FEV} 1<80 \%$ predicted. COPD hospitalisation: International Classification of Diseases (ICD)-8 491-492 or ICD-10 J41-J44. Odds ratios for spirometrically defined COPD are by logistic regression. The models allowed for age, sex, pack-yrs, passive smoking, occupational dust and fumes, education, and occupation. p-values for SP-B genotypes and genotype combinations were by test for trends and ANOVA, respectively. Numbers for the analysis of spirometrically defined COPD are slightly less than for COPD hospitalisation, because not all individuals had spirometry performed.

on lung function are in accordance with a previous study on healthy males, which showed no association between rs1130866 and FEV1 \% pred or FVC \% pred [21].

The $S P-B$ promoter variations rs2077079 and rs3024791 have previously, in studies with $<400$ participants, been associated with severity of airway obstruction and exacerbations in COPD patients, but not with risk of developing COPD $[3,16]$. Both polymorphisms alter transcription of the $S P-B$ gene and may be associated with altered levels of SP-B in the airways. We did not find any overall association of the two promoter variants with lung function, risk of COPD or COPD hospitalisation. We have previously shown that partial SP-B deficiency due to the rare 121ins 2 mutation is associated with reduced pulmonary function and increased risk of COPD among smokers [4]. However, when stratifying our data for smoking status, none of the two promoter polymorphisms were associated with reduced pulmonary function or risk of COPD among smokers.

It has been suggested that the rs1130866 polymorphism may require other interacting factors to alter the pulmonary phenotype. GuO et al. [3] suggested that gene-gene and geneenvironment interactions may be important, and HERSH et al. [18] found rs113086 to be associated with COPD only in a statistical model with the presence of a gene-smoking interaction term. To mimic gene-gene interactions and gene-environment interactions, we assessed lung function and calculated COPD risk estimates for combinations of rs1130866, rs2077079 and rs3024791 stratified by smoking status. When stratifying our analyses by smoking status, we were unable to show association of $S P-B$ genotype combinations with lung function and risk of COPD among smokers. Among never-smokers, we did find an association between certain genotype combinations and lung function or COPD. However, these results may not be biologically plausible and they could not be confirmed using lung function or the other COPD end-point of the study. If correction for 60 and 30 multiple comparisons was performed for the analyses of $S P-B$ combinations and lung function (fig. 2), and SP-B combinations and COPD (fig. 4), respectively, none of the results observed among never-smokers would be of statistical significance. We, therefore, interpret these findings 
as likely spurious results. The lack of overall association of $S P-B$ polymorphisms with pulmonary function and disease in our study is supported by recent genome-wide association studies and a novel Dutch population/case-control study [22-24].

As variations in $S P-B$ have been associated with respiratory diseases other than COPD [9, 10, 25], we also tested for association between the three polymorphisms and risk of common pulmonary diseases, such as asthma, pneumonia and lung cancer. We found no consistent association between $S P-B$ genotypes and any of the lung diseases we examined, except for asthma. As in previous reports on SP-C [26, 27], a protein related to SP-B, we did find an association between $S P-B$ rs3024791 rare versus common homozygosity and asthma. We could not confirm this result when analysing other $S P-B$ genotypes, and further studies will be needed to conclusively determine whether the $S P-B$ rs3024791 AA genotype is associated with increased asthma risk.

Some misclassification of spirometrically defined COPD is possible, and this could limit the subgroup analysis according to GOLD classification. The pulmonary function tests used to define COPD were not performed post-bronchodilator due to the large number of subjects included in the study and our limited funds. However, excluding individuals with asthma from this definition did not substantially alter our results. Furthermore, in the 1991-1994 survey those individuals who had FEV1/FVC $<0.7$ had post-bronchodilator spirometry performed. If analyses on pulmonary function and COPD were confined to this subgroup, we found no difference in FEV1 \% pred, FEV1/FVC or COPD prevalences according to $S P-B$ genotype or genotype combination. Lack of association between any of the $S P-B$ polymorphisms with pulmonary function or disease, as opposed to previous findings, could be due to different genotype frequencies among the studied populations. HERSH et al. [18] reported a carrier frequency for the rs1130866 T allele of 0.44 for controls and 0.46 for cases. We found the overall frequency of the $\mathrm{T}$ allele to be 0.54 . FOREMAN et al. [16] reported carrier frequencies for rs3024791 GG, AG and AA genotypes of $0.73,0.25$ and 0.02 , respectively. Our results were very similar $(0.75,0.23$ and 0.02 , respectively). Gou et al. [3] reported a carrier frequency for the rs1130866 C allele of 0.67 for controls and 0.82 for cases. We found an overall carrier frequency for the rs $1130866 \mathrm{C}$ allele of 0.71 . Bias caused by investigator knowledge of disease or risk factor status seems unlikely, because our sample was selected from the general population and genotyping of our sample was performed without investigator knowledge of disease status or lung function test results.

In conclusion, we find, with significant power, that three common functional polymorphisms in the $S P-B$ gene are not associated with reduced lung function or risk of COPD in the Danish general population overall or among smokers. This makes it unlikely that these genetic variants will have a role in personalised medicine. Though our results are based on individuals of Danish/European descent, these polymorphisms are prevalent in many populations and our results may apply to other parts of the World.

\section{STATEMENT OF INTEREST}

None declared.

\section{REFERENCES}

1 Murray CJ, Lopez AD. Alternative projections of mortality and disability by cause 1990-2020: Global Burden of Disease Study. Lancet 1997; 349: 1498-1504.

2 Hersh CP, DeMeo DL, Lazarus R, et al. Genetic association analysis of functional impairment in chronic obstructive pulmonary disease. Am J Respir Crit Care Med 2006; 173: 977-984.

3 Guo X, Lin HM, Lin Z, et al. Surfactant protein gene A, B, and D marker alleles in chronic obstructive pulmonary disease of a Mexican population. Eur Respir J 2001; 18: 482-490.

4 Baekvad-Hansen M, Dahl M, Tybjaerg-Hansen A, et al. Surfactant protein-B 121ins2 heterozygosity, reduced pulmonary function, and chronic obstructive pulmonary disease in smokers. Am J Respir Crit Care Med 2010; 181: 17-20.

5 Andreeva AV, Kutuzov MA, Voyno-Yasenetskaya TA. Regulation of surfactant secretion in alveolar type II cells. Am J Physiol Lung Cell Mol Physiol 2007; 293: L259-L271.

6 Dietl P, Haller T. Exocytosis of lung surfactant: from the secretory vesicle to the air-liquid interface. Annu Rev Physiol 2005; 67: 595-621.

7 Hawgood S. Surfactant protein B: structure and function. Biol Neonate 2004; 85: 285-289.

8 Wilder MA. Surfactant protein B deficiency in infants with respiratory failure. J Perinat Neonatal Nurs 2004; 18: 61-67.

9 Floros J, Lin HM, Garcia A, et al. Surfactant protein genetic marker alleles identify a subgroup of tuberculosis in a Mexican population. J Infect Dis 2000; 182: 1473-1478.

10 Lin Z, Pearson C, Chinchilli V, et al. Polymorphisms of human SP-A, SP-B, and SP-D genes: association of SP-B Thr131Ile with ARDS. Clin Genet 2000; 58: 181-191.

11 Quasney MW, Waterer GW, Dahmer MK, et al. Association between surfactant protein $B+1580$ polymorphism and the risk of respiratory failure in adults with community-acquired pneumonia. Crit Care Med 2004; 32: 1115-1119.

12 Floros J, Veletza SV, Kotikalapudi P, et al. Dinucleotide repeats in the human surfactant protein-B gene and respiratory-distress syndrome. Biochem J 1995; 305: 583-590.

13 Seifart C, Plagens A, Brodje D, et al. Surfactant protein B intron 4 variation in German patients with COPD and acute respiratory failure. Dis Markers 2002; 18: 129-136.

14 Hu R, Xu Y, Zhang Z. Surfactant protein B 1580 polymorphism is associated with susceptibility to chronic obstructive pulmonary disease in Chinese Han population. J Huazhong Univ Sci Technolog Med Sci 2004; 24: 216-218.

15 Steagall WK, Lin JP, Moss J. The C/A(-18) polymorphism in the surfactant protein $\mathrm{B}$ gene influences transcription and protein levels of surfactant protein B. Am J Physiol Lung Cell Mol Physiol 2007; 292: L448-L453.

16 Foreman MG, DeMeo DL, Hersh CP, et al. Polymorphic variation in surfactant protein B is associated with COPD exacerbations. Eur Respir J 2008; 32: 938-944.

17 Thomas KH, Meyn P, Suttorp N. Single nucleotide polymorphism in 5'-flanking region reduces transcription of surfactant protein B gene in H441 cells. Am J Physiol Lung Cell Mol Physiol 2006; 291: L386-L390.

18 Hersh CP, DeMeo DL, Lange C, et al. Attempted replication of reported chronic obstructive pulmonary disease candidate gene associations. Am J Respir Cell Mol Biol 2005; 33: 71-78.

19 Dahl M, Bowler RP, Juul K, et al. Superoxide dismutase 3 polymorphism associated with reduced lung function in two large populations. Am J Respir Crit Care Med 2008; 178: 906-912.

20 Lee J, Nordestgaard BG, Dahl M. Elevated ACE activity is not associated with asthma, COPD, and COPD co-morbidity. Respir Med 2009; 103: 1286-1292.

21 Raleigh SM, Davies BM, Cleal D, et al. No association between coding polymorphism within Exon 4 of the human surfactant protein B gene and pulmonary function in healthy men. J Physiol Sci 2007; 57: 199-202. 
22 Wilk JB, Chen TH, Gottlieb DJ, et al. A genome-wide association study of pulmonary function measures in the Framingham Heart Study. PLoS Genet 2009; 5: e1000429.

23 Pillai SG, Ge D, Zhu G, et al. A genome-wide association study in chronic obstructive pulmonary disease (COPD): identification of two major susceptibility loci. PLoS Genet 2009; 5: e1000421.

24 van Diemen CC, Postma DS, Aulchenko YS, et al. Novel strategy to identify genetic risk factors for COPD severity: a genetic isolate. Eur Respir J 2010; 35: 768-775.
25 Seifart C, Seifart U, Plagens A, et al. Surfactant protein B gene variations enhance susceptibility to squamous cell carcinoma of the lung in German patients. Br J Cancer 2002; 87: 212-217.

26 Erpenbeck VJ, Schmidt R, Gunther A, et al. Surfactant protein levels in bronchoalveolar challenge in patients with asthma. Allergy 2006; 61: 598-604.

27 Baekvad-Hansen M, Nordestgaard BG, Tybjaerg-Hansen A, et al. Two novel mutations in surfactant protein-C, lung function and obstructive lung disease. Respir Med 2010; 104: 418-425. 